\title{
Green synthesis of controlled size gold and silver nanoparticles using antioxidant as capping and reducing agent
}

\author{
Musammir Khan $^{\mathrm{a}, *}$, Fawad Ahmad ${ }^{\mathrm{a}}$, Janne T. Koivisto ${ }^{\mathrm{b}, \mathrm{c}}$, Minna Kellomäki ${ }^{\mathrm{b}}$ \\ ${ }^{a}$ Department of Chemistry, University of Wah, Quaid Avenue, Wah Cantt., Rawalpindi, Punjab, Pakistan \\ ${ }^{\mathrm{b}}$ Biomaterials and Tissue Engineering Group, BioMediTech Institute, Faculty of Medicine and Health Technology, Tampere University, Korkeakoulunkatu 10, 33720 \\ Tampere, Finland \\ ${ }^{\mathrm{c}}$ Microelectronics Research Unit, Faculty of Information Technology and Electrical Engineering, University of Oulu, PL 4500, 90570 Oulu, Finland
}

\section{A R T I C L E I N F O}

\section{Keywords:}

Green synthesis

Antioxidant

Metallic nanoparticles

Cytotoxicity

\begin{abstract}
A B S T R A C T
Here silver (GA-AgNPs) and gold nanoparticles (GA-AuNPs) were synthesized by an ecofriendly method using antioxidant, gallic acid (GA) simultaneously as capping and reducing agent. Their size ranged between $15 \mathrm{~nm}$ and $80 \mathrm{~nm}$, which was controlled or dependent on the feeding ratio of GA. There was a linear relationship between the particle sizes versus the GA content. Moreover, they indicated a negative zeta potential value, which was decreased from $-33 \mathrm{mV}$ to $-40 \mathrm{mV}$ for AgNPs and from $-36 \mathrm{mV}$ to $-42 \mathrm{mV}$ for AuNPs after the addition of GA . In a typical PEGylation reaction of them, resulted into a large increase in the particle size as well as zeta potential value. The cytotoxicity assay against human fibroblast cell line (WI-38) for $24 \mathrm{~h}$ and 3-days showed that they were completely safe toward these cells. Therefore, these engineered NPs could be considered promising supplementary targeting materials in future.
\end{abstract}

\section{Introduction}

Over the past decades there has been extensive interest in developing metal nanoparticles (MNPs) as effective devices for catalysis [1-3], optoelectronics [4,5], surface enhanced Raman scattering [6,7], biological labeling [8], chemical [9] or biosensors [10] and drug delivery [11,12] applications [13]. Most of these properties depend on their size, shape, crystalline structure and surface functionalization [14-16].

Generally these NPs are hybrid material consisting of inorganic core typically surrounded by an organic monolayer. During the fundamental interaction with the environment, the monolayer usually determines the chemical behavior, while the core governs the physical features of the NPs [17]. These engineered NPs have smaller sizes and hence possess greater specific surface area or surface-to-volume ratio which could results in their enhanced reactivity and sensitivity effect in comparison to their bulk counterpart $[18,19]$.

Selection of appropriate biological entity for the preparation of the monolayer in term of novelty, ecofriendly nature and availability can be considered as third generation nanobiotechnological approach [20]. More importantly, there has been an extensive effort to develop ecobenign and nontoxic method for the synthesis of noble MNPs of gold, silver, palladium, selenium etc. Plant extracts are used as reducing agents, which contains enzymes and natural phytochemicals such as terpenoids, flavonoids and phenols. In addition isolated carbohydrates, proteins, amino acids as well as bacteria, fungi, yeast and certain algae can also work as rapid, simple and biocompatible reducing agents [21]. From the broad spectra of nanoparticles, silver nanoparticles (AgNPs) has been exploited in several fields such as medicine, dentals, textiles and waste water treatment or remediation due to their antibacterial and catalytic degradation of soluble pollutants (i.e. 4-nitrophenol or methylene blue), low cytotoxicity, low volatility and high thermal stability [22-24]. On the other hand the size dependent physicochemical and biological properties and the unique optical nature arising from surface Plasmon oscillation of free electrons of gold nanoparticles (AuNPs) have been proposed for important biomedical areas such as drug or gene delivery, cancer treatment, photothermal therapy of tumor and biological imaging $[25,26]$. It is worthy to note that smaller size AuNPs (i.e. size below 4-5 nm) were identified as potential toxic because of their cellular penetration and DNA binding [27].

Among several synthetic methods used for nanoparticles, the popular examples are laser evaporation and chemical reduction. However, the first one is limited by its costly nature and low yield of production, while the later one has drawback of leaving behind toxic chemicals adsorbed onto the NPs surface, which diminishes its value for medical applications [28]. The reduction of tetrachloroauric acid $\left(\mathrm{HAuCl}_{4}\right)$ in

\footnotetext{
* Corresponding author.

E-mail address: musammir.khan@uow.edu.pk (M. Khan).
} 
aqueous media using trisodium citrate $\left(\mathrm{Na}_{3} \mathrm{Ct}\right)$ is well established protocol referred to as "Turkvich synthesis" [29] to prepare the colloidal stable well-dispersed negatively charge NPs. Similarly, the silver nitrate $\left(\mathrm{AgNO}_{3}\right)$ using two reductants $\mathrm{Na}_{3} \mathrm{Ct}$ and tannic acid resulted in the stable monodisperse NPs. The importance of this method could be attributed to the loosely bound nature of $\mathrm{Na}_{3} \mathrm{Ct}$ to the particle, which create accessible surface especially for biomolecule functionalization [30-32].

Gallic acid (GA) is a phenolic compound having radical scavenging ability, preventive and therapeutic effect against certain oxidative stress related diseases such as cardiovascular, cancer, neurodegenerative and ageing. Due to these pharmacological activities, it could be considered for developing new drugs in the future [33]. Au and Ag NPs conjugated with GA have potential medical applications such antibacterial activity [34]. Gallic acid capped AgNPs (GA-AgNPs) have potential application as electrochemical sensors or calorimetric and spectroscopic probes [35,36].

In view of the biological importance of the natural antioxidant, GA, it was used in combination with $\mathrm{Na}_{3} \mathrm{Ct}$ as reducing and capping/stabilizing agent to synthesize GA-AgNPs and GA-AuNPs. Keeping other experimental parameters constant, the effect of GA concentration on the particle size and size distribution, surface charge and surface modification with polyethylene glycol (PEG) have been evaluated. The in-vitro biocompatibility of these NPs was tested against human fibroblast cells WI-38.

\section{Materials and methods}

\subsection{Materials}

All the chemicals were purchased from Sigma Aldrich i.e. $\mathrm{AgNO}_{3}$ (99\%), $\mathrm{HAuCl}_{4}(99 \%)$, trisodium citrate dihydrate $\left(\mathrm{Na}_{3} \mathrm{Ct} .2 \mathrm{H}_{2} \mathrm{O}\right)(99 \%)$, gallic acid (GA) (99\%), otherwise specifically mentioned. PEG-5 k 95\% was bought from JenKem Technology USA.

\subsection{Methods}

\subsubsection{Preparation of nanoparticles}

The GA capped AgNPs (GA-AgNPs) was prepared using our previous protocol [16] with slight modification i.e. different amount of GA $(0.156,0.313,0.625,1.25,2.5,5.0 \mathrm{mM})$ were separately dissolved in $100 \mathrm{~mL}$ deionized (DI) water in a $250 \mathrm{~mL}$ flask, then constant amount of $\mathrm{Na}_{3} \mathrm{Ct}(5 \mathrm{mM})$ was added to each flask under constant stirring and the mixture was heated until boiling. Then constant amount of $\mathrm{AgNO}_{3}$ ( $1 \mathrm{~mL}$ of $25 \mathrm{mM}$ ) was injected to each boiling solution and stirred until the color changes from colorless to bright/deep yellow.

For GA-AuNPs preparation, constant amount of $\mathrm{Na}_{3} \mathrm{Ct}(2.5 \mathrm{mM}$ in $150 \mathrm{~mL}$ DI water) and varying amount of GA $(0,0.625,1.25,2.5 \mathrm{mM})$ were added to each separate solution in $250 \mathrm{~mL}$ flasks and heated under constant stirring until boiling. Then at boiling the gold salt $\left(\mathrm{HAuCl}_{4}\right)(25 \mathrm{mM})$ dissolved in $1 \mathrm{~mL}$ DI water was added to the above solution. The mixture was heated continuously until color change from yellow to ruby red.

PEGylation of the NPs was performed using heterofunctional PEG$5 \mathrm{k}$ (i.e. one end hydroxyl and another methoxy group terminated) using EDC reaction for $24 \mathrm{~h}$. The NPs solution was purified by centrifugation and then resuspended in DI and ultrasonicated for 2 min to prevent aggregation.

\subsubsection{Characterization}

The UV/visible spectra of NPs solution was measured using double beam spectrophotometer (Shimadzu UV-1900). Distilled water was used as reference standard.

Particle size, size distribution and zeta potential value was measured using Zetasizer Nano ZS-Malvern Panalytical (DLS).

\subsubsection{In-vitro cell viability assay}

The in-vitro assay was performed with the commercial human fibroblast cell line WI-38 (Culture Collections, Public Health England, UK) for $24 \mathrm{~h}$ and 3 days according to our previous protocol [37].

\section{Results and discussion}

\subsection{Synthesis of GA-capped NPs}

The synthesis of GA capped metallic GA-AgNPs and GA-AuNPs is convenient and straightforward using the previous standard protocol in combination with the green chemistry approach to tailor their physicochemical properties. The current synthetic method is rapid and highly reproducible using bioactive molecule GA as reducing and capping agent [38]. It was previously identified that GA concentration is crucial for manipulating the size of these NPs owing to the presence of its bifunctional nature. At normal $\mathrm{pH}$ condition (proposed $\mathrm{pH}=\sim 7$ ), it undergoes a 2-electron oxidation process of its two hydroxyl group forming quinone and at this $\mathrm{pH}$ is also present in ionized form thus enhancing the interaction with the NPs surface and stabilizing through negative carboxyl group (shown in Scheme 1) [39].

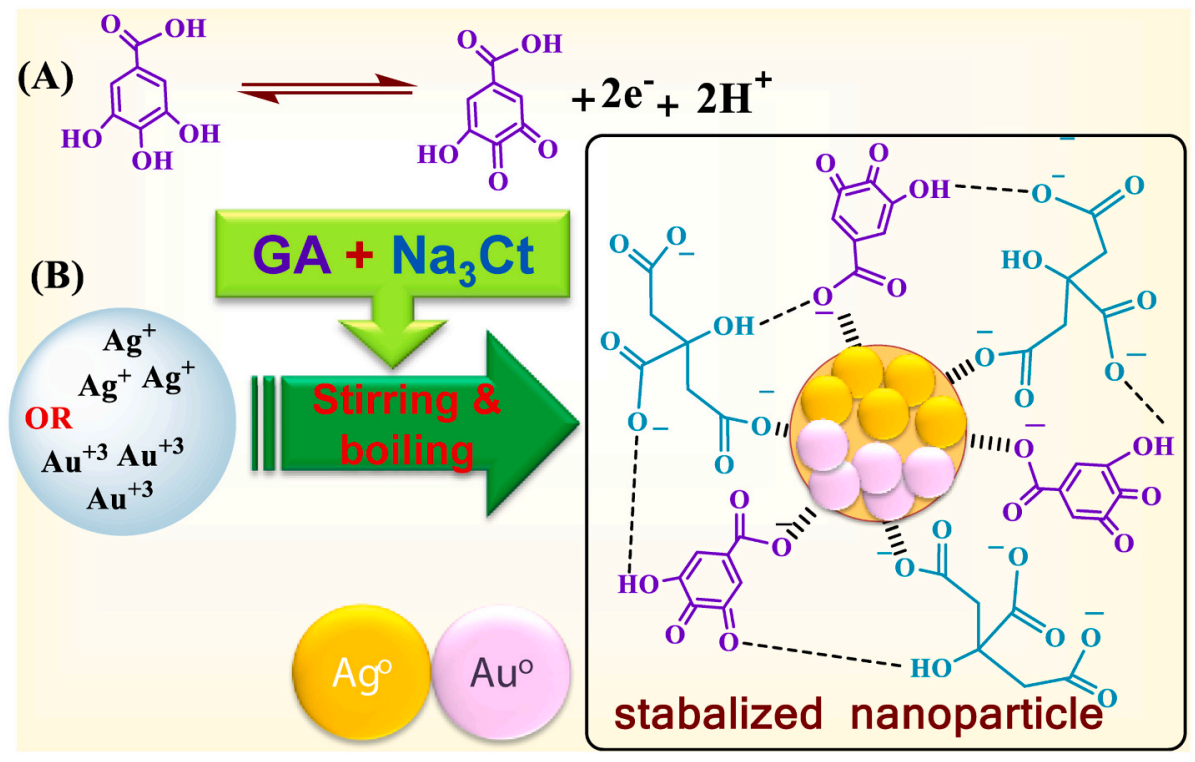

Scheme 1. Preparation of AgNPs and AuNPs using gallic acid (GA) and trisodium citrate $\left(\mathrm{Na}_{3} \mathrm{Ct}\right)$ as capping and reducing agents; (A) GA undergo a 2electron reduction process, (B) preparation of GAAgNPs and GA-AuNPs. The hatched lines (in inset box) show the interaction of GA and trisodium citrate $\left(\mathrm{Na}_{3} \mathrm{Ct}\right)$ with NPs surface, while the narrow dashed line represent H-bonding . 


\subsection{UV-visible spectroscopy}

The UV/visible spectra (Fig. 1) of GA-AgNPs (A) and GA-AuNPs (B) using different GA content, showed a clear red-shift as well as broadening in the surface Plasmon resonance peak (SPR) with increasing the concentration of GA. This SPR shift was linear in the studied GA concentration $(0.156-5.00 \mathrm{mM})$ range, with $\lambda_{\max }$ from $404 \mathrm{~nm}$ to $441 \mathrm{~nm}$ for GA-AgNPs and (0.625-2.5 mM) for GA-AuNPs with $\lambda_{\max }$ shift from $518 \mathrm{~nm}$ to $531 \mathrm{~nm}$. The color of GA-AgNPs solution (inset box) was changed from deep yellow to light yellow with the increase with GA, while that of AuNPs was shifted from red toward dark purple, both of which indicated an increase in particle size. Such kind of characteristic SPR peaks in the visible wavelength region with a red shift and peak broadening upon increasing the GA concentration was also observed by Park et al. for the GA conjugated AgNPs and AuNPs. This study indicated that the size of the NPs can be fine-tuned by varying the GA concentration $[34,40,41]$.

\subsection{Nanosizer/dynamic light scattering}

The Nanosizer/DLS analysis of these NPs indicated that for both kinds of particle the size was linearly increased with the GA concentration (Table 1). In case of AgNPs this size increase was from $35 \mathrm{~nm}$
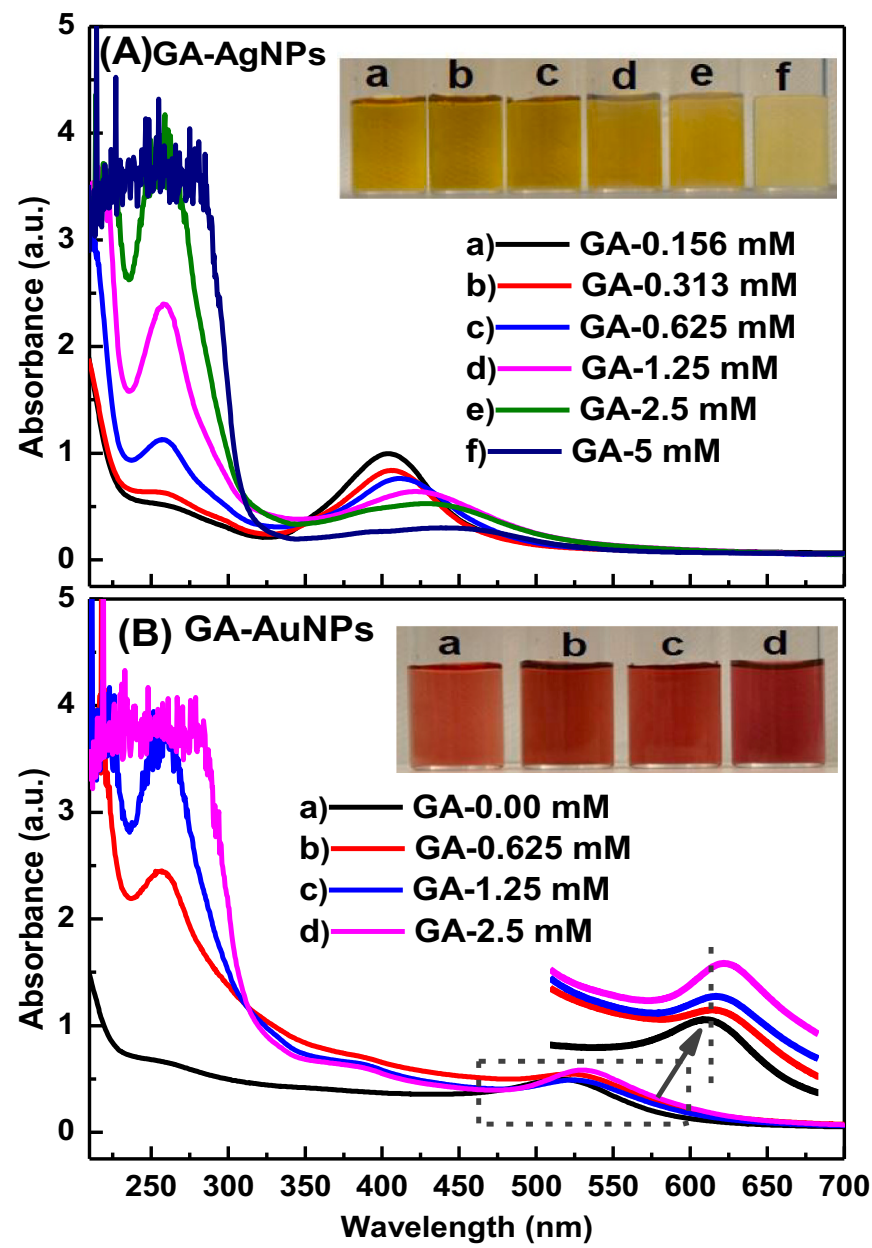

Fig. 1. UV-visible spectra of gallic acid (GA) capped nanoparticles, inset with color photos (data) show different concentrations of GA: A) Silver nanoparticles (GA-AgNPs, GA $=0.156 \mathrm{mM}-5 \mathrm{mM}$ ), B) Gold nanoparticles (GA-AuNPs; $\mathrm{GA}=0 \mathrm{mM}-2.5 \mathrm{mM}$ ). (For interpretation of the references to color in this figure legend, the reader is referred to the web version of this article.)
Table 1

UV/Vis and DLS results of GA-capped silver and gold NPs showing SPR peaks, average particle size and zeta potential values as a function of GA concentrations.

\begin{tabular}{lllll}
\hline Sample ID & $\begin{array}{l}\text { GA content } \\
(\mathrm{mM})\end{array}$ & $\begin{array}{l}\text { SPR peak } \\
(\mathrm{nm})\end{array}$ & $\begin{array}{l}\text { Particle size } \\
(\mathrm{nm}) \pm 1\end{array}$ & $\begin{array}{l}\text { Zeta potential } \\
(\mathrm{mV})\end{array}$ \\
\hline GA-AgNPs & 0.156 & 404 & 35 & -33 \\
& 0.313 & 407 & 38 & -34 \\
& 0.6125 & 409 & 50 & -34 \\
& 1.25 & 422 & 78 & -40 \\
& 2.5 & 430 & 75 & -40 \\
GA-AuNPs & 5.0 & 441 & 79 & -38 \\
& 0.6125 & 518 & 18 & -36 \\
& 1.25 & 525 & 26 & -42 \\
& 2.5 & 527 & 32 & -36 \\
\end{tabular}

to $79 \mathrm{~nm}$ as the GA content was increased from $0.156 \mathrm{mM}$ to $5 \mathrm{mM}$. This size increase for AuNPs was from $18 \mathrm{~nm}$ to $59 \mathrm{~nm}$ in the studied concentration range of GA from $0 \mathrm{mM}$ to $2.5 \mathrm{mM}$.

The zeta potential value of AgNPs decreased from $-33 \mathrm{mV}$ to $-40 \mathrm{mV}$ upon increasing the GA content, while in case of AuNPs the zeta potential (ZP) value first decreased from $-36 \mathrm{mV}$ to $-42 \mathrm{mV}$ and then increased to $-36 \mathrm{mV}$ (see Table 1). This shows that the higher negative charge still persists on the NPs surface due to the presence of negative charged carboxyl group attached to the nanoparticle surface, which provided stability resulted from the electrostatic repulsion of NPs in the solution. In the previous report of AgNPs based on pure GA as reducing and capping agent indicated a higher $\mathrm{ZP}$ value of $-10 \mathrm{mV}$ [42], while in another report using a combination of $\mathrm{NaBH}_{4}$ and GA as reducing agent, showed $\mathrm{ZP}$ value of $-34 \mathrm{mV}$, where the attachment mechanism of pure GA to the NPs surface was based on chelate formation through hydroxyl group [35].

The DLS results (Fig. 2) shows that both kinds of GA-NPs behaved a broad size distribution upon increasing GA concentration, while keeping the other experimental parameters (i.e. $\mathrm{Na}_{3} \mathrm{Ct}$ and salt conc., Temp) constant In case of GA-AgNPs this size was linearly increased as the GA content was raised from $0.156 \mathrm{mM}$ to $1.25 \mathrm{mM}$ and further there was no appreciable improvement or change in the size, which could be considered the saturation/critical point for the reductant concentration. Moreover, there was no color change (or no reduction) when no GA was used (i.e. $\mathrm{GA}=0 \mathrm{mM}$ ) in case of AgNPs.

Note, from both UV/visible spectra (Fig. 1) and DLS analysis (Table 1 plus Fig. 2), it is cleared that the reproducible and optimal conditions for the synthesis of these GA-NPs were; for GA-AgNPs (Figs. 1 and $2 \mathrm{~A}$ ) was (GA $=0.625 \mathrm{mM}$, average size $=50 \pm 1 \mathrm{~nm}$, $\mathrm{SPR}=409 \mathrm{~nm}$, Zeta $=-34 \mathrm{mV}$ ), while for GA-AuNPs (Figs. 1 and $2 \mathrm{~B}$ ) was $(\mathrm{GA}=1.25 \mathrm{mM}$, average size $=32 \pm 1 \mathrm{~nm}, \mathrm{SPR}=527 \mathrm{~nm}$, Zeta $=-36 \mathrm{mV}$ ).

The kinetics of GA-NPs corona by autocorrelation or correlation coefficient using DLS (Fig. 3) showed that the size of GA capped NPs (GA-AgNPs \& GA-AuNPs) increased due to the surface adsorption of GA and monolayer formation or corona of the NPs. The autocorrelation function show broadening as indicated by increase in autocorrelation time with increase of GA concentration in the solution. This shows that a larger corona size will decrease the diffusion time $(\tau \mu s)$ and shifted the autocorrelation function to longer times [43]. The diffusion coefficient (D) in these systems can be calculated using Stokes-Einstein Eq. (1) [44]:

$\mathrm{D}=\frac{k_{B} T}{3 \pi \eta d_{n}}$

Where $k_{B}$ is Boltzmann's constant, $T$ absolute temperature, $\eta$ viscosity of solvent and $d_{n}$ is measured hydrodynamic diameter of the NPs. 

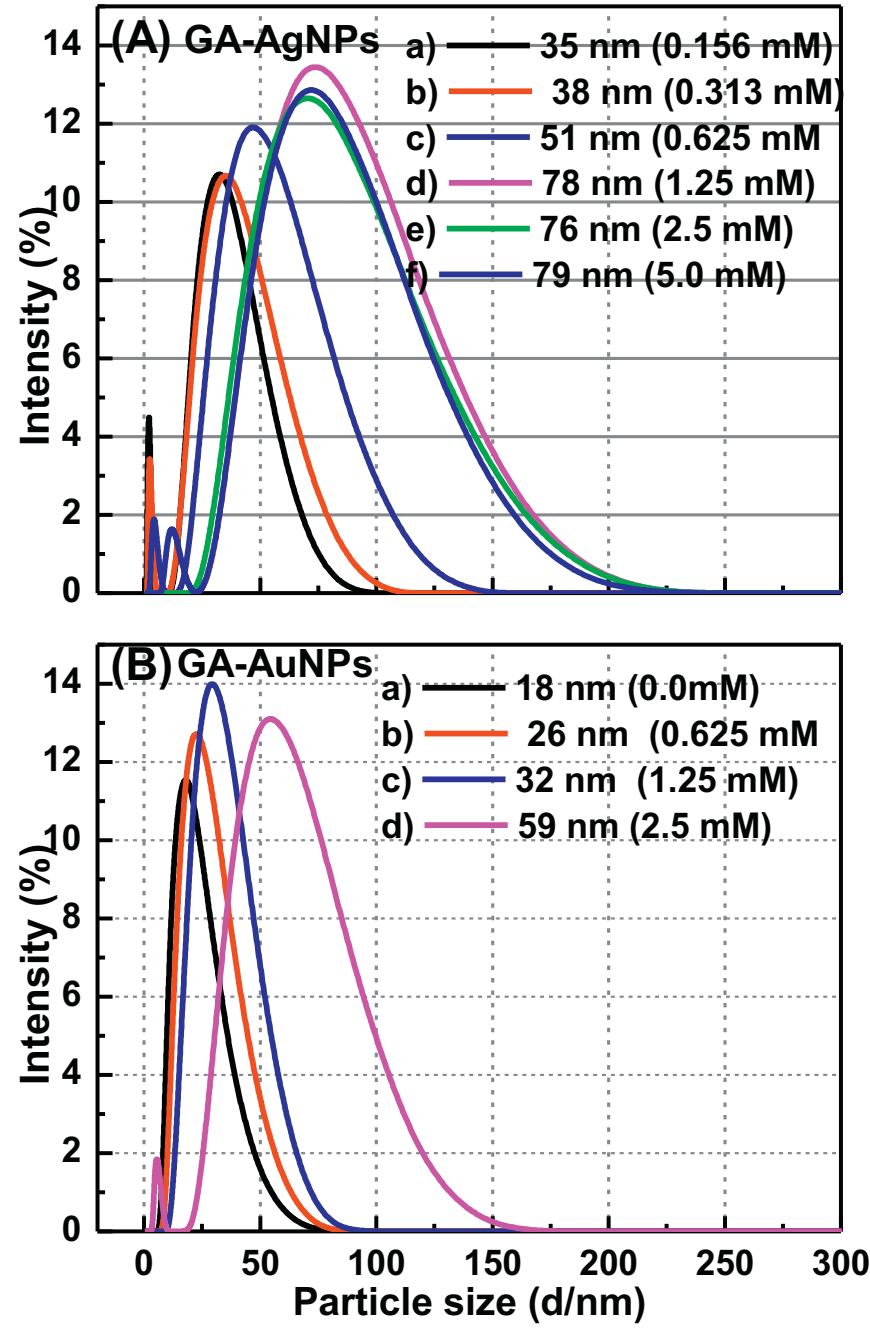

Fig. 2. Particle size and size distribution of gallic acid (GA) capped nanoparticles measured by DLS A) GA-AgNPs using different molar ratios of GA $(\mathrm{GA}=0.156 \mathrm{mM}-5 \mathrm{mM}), \mathrm{B})$ GA-AuNPs (with $\mathrm{GA}=0 \mathrm{mM}-2.5 \mathrm{mM}$ ).

\subsection{PEGylation of GA-NPs}

The PEGylation of the GA-NPs was performed by taking the advantage of the negatively charged shell, which consists of plenty of carboxyl group (of $\mathrm{Na}_{3} \mathrm{Ct}$ : Scheme-1), using monohydroxy-PEG by a single step EDC reaction. Furthermore, the effect of surface modification (stealth coating) on overall performance of the NPs, such as on the particle size/distribution, surface charge effect and SPR position was investigated using the same optimal conditions GA-NPs sample from both GA-AuNPs and GA-AgNPs. The UV/visible spectral analysis (Fig. 4) of GA-AgNPs (size $=50 \mathrm{~nm}, \mathrm{GA}=0.625 \mathrm{mM}$ ) before and after PEGylation (of the as prepared NPs) indicated a clear red shift (bathochromic effect) with SPR shift from $409 \mathrm{~nm}$ to $416 \mathrm{~nm}$, while average particle size (Table 1) corresponding to the UV SPR peaks was shifted from $50 \mathrm{~nm}$ to $177 \mathrm{~nm}$, with an increase in zeta potential from -34 to $-11 \mathrm{mV}$, respectively. On the other hand, in case of GA-AuNPs ( size $=32 \mathrm{~nm}, \mathrm{GA}=1.25 \mathrm{mM})$, the UV SPR peaks was shifted from $531 \mathrm{~nm}$ (before PEGylation) to $542 \mathrm{~nm}$ (after PEGylation). Furthermore, the average particle size varied from $32 \pm 1 \mathrm{~nm}$ to 170 and the zeta potential value was increased from -36 to $-23 \mathrm{mV}$ after PEGylation. This indicated that PEGylation has profound effect on the physicochemical properties of these GA-NPs.

In order to study the effect of various physicochemical parameters on the particle size, size distribution and surface charge, a
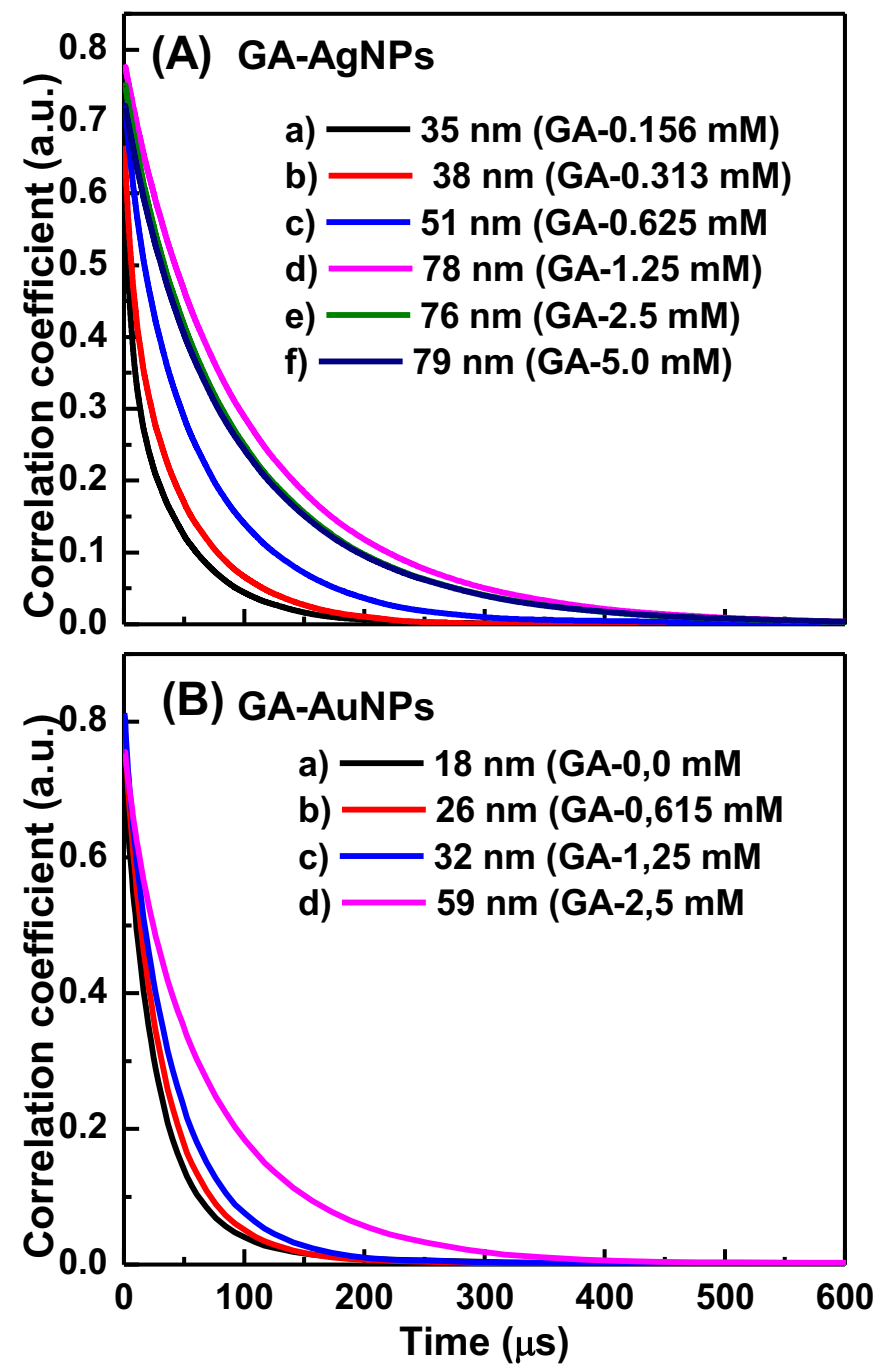

Fig. 3. Autocorrelation function or correlation coefficient measured by DLS: A) GA-AgNPs at different molar ratios of GA (GA $=0.156 \mathrm{mM}-5 \mathrm{mM}$ ), B) GAAuNPs with GA $=0 \mathrm{mM}-2.5 \mathrm{mM}$.

representative NPs (AuNPs-GA: GA $=1.25$, size $=32 \mathrm{~nm}$ ) sample was subjected to chemical modification and mechanical steps (Fig. 5), such as purification (centrifugation), ultrasonication and PEGylation (AuNPs-PEG). The AuNPs-PEG was centrifuged (separated/purified) and then finally ultrasonicated. This process clearly shows that after the first ultrasonication for about 2 min the original particle had a broad size distribution (Fig. 5A), with a size increase from $32 \mathrm{~nm}$ to $70 \mathrm{~nm}$ and a slight rise in zeta potential value from -36 to $-32 \mathrm{mV}$. The phenomenon might be attributed to the interionic interaction of carboxyl group of $\mathrm{Na}_{3} \mathrm{Ct}$ with hydroxyl moiety of the GA as well as the reorganization of capping agents. After PEGylation of the same NPs, there was a huge increase in particle size i.e. from $32 \mathrm{~nm}$ to $170 \mathrm{~nm}$, with a broad size distribution and also with a prominent increase in zeta potential from -32 to $-15 \mathrm{mV}$. After ultrasonication for about $2 \mathrm{~min}$, however, the particle size again decreased to $108 \mathrm{~nm}$, showed a narrow size distribution, while the zeta potential value became lowered to $-23 \mathrm{mV}$ (Fig. 5B). Thus the reduction in particle size as well as zeta potential values in the final purification step of the PEGylated NPs facilitated the removal of excess reagents (PEG, catalyst) and thus prevented the aggregation due to interparticle interactions.

In comparison to the previous green chemistry protocols, using plant/microbes extracts as reducing and capping agents is vital to remediate the toxicity on one hand, but the size control, modulation and 


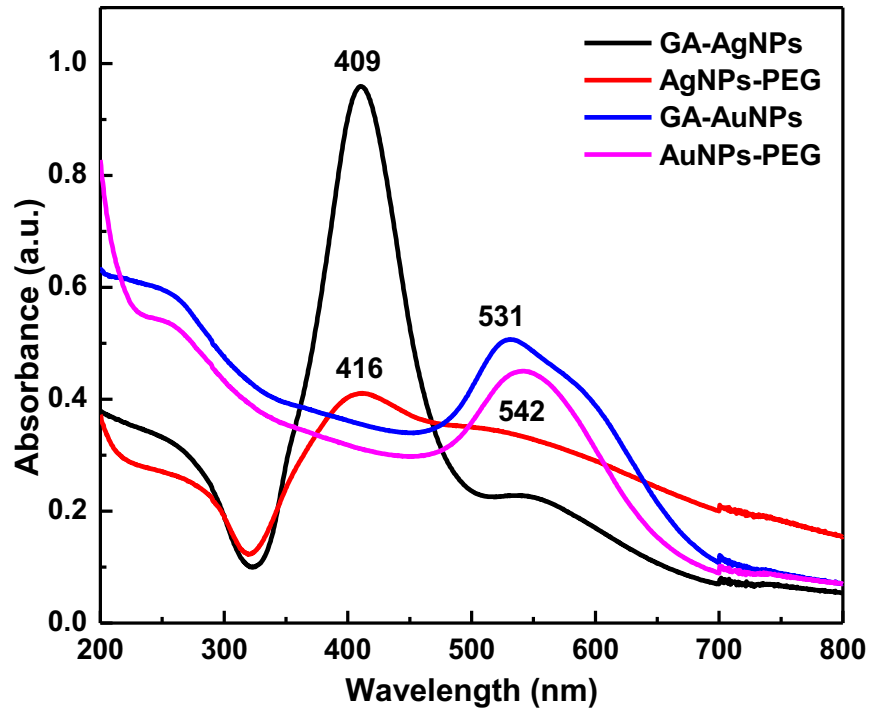

Fig. 4. UV-visible spectra of GA capped silver (GA-AgNPs) and gold nanoparticles (GA-AuNPs) before and after PEGylation. (For interpretation of the references to color in this figure legend, the reader is referred to the web version of this article.)
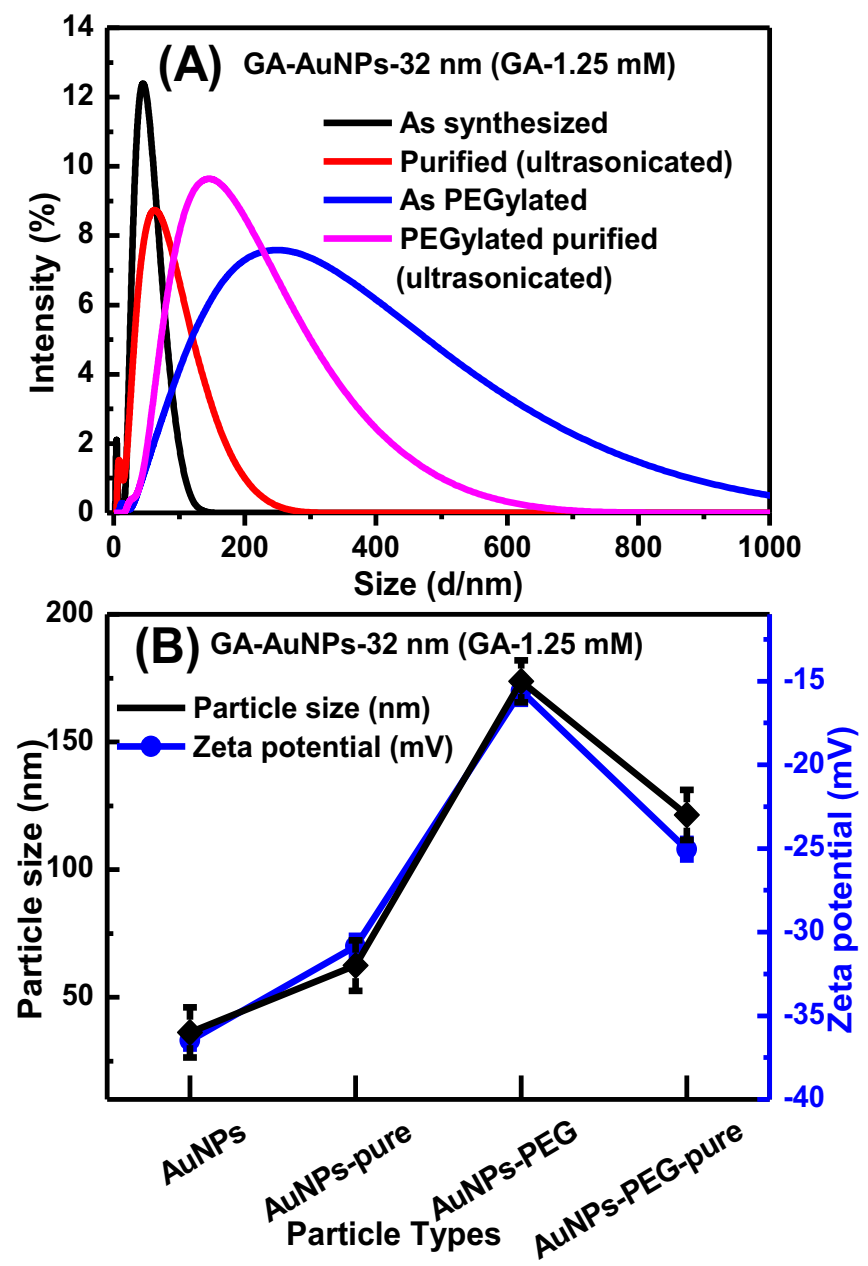

Fig. 5. DLS of a typical GA-AuNPs (GA-1.25 mM, size $=32 \pm 1 \mathrm{~nm}$ ) showing; (A) particle size distribution, (B) variation in particle size ( $\mathrm{nm}$ ) and zeta potential values before and after PEGylation. particle dispersity is a great challenge on the other hand $[45,46]$. In spite of attaining desired size/shape with reasonably good properties as claimed using previous green synthesis, it seem rather unclear how theparticle size or surface charge is systematically controlled as a function of specific reductant concentration in that particular plant extract $[22,25,26]$. Therefore, our protocol could provide a useful and systematic way to overcome this particular challenge of size control or dispersity as well as surface charge as a function of only a single reductant concentration variation in the feeding solution.

The size, surface morphology and the mean size distribution of the GA capped NPs of silver (GA-AgNPs (A)) and gold (GA-AuNPs (B)) was confirmed by SEM analysis (Fig. 6). These typical SEM images indicated that the particles were spherical in morphology and with monodisperse size distribution. The mean particle size of them was $35 \mathrm{~nm}$ for AgNPsGA and $24 \mathrm{~nm}$ for GA-AuNPs. Note, we have comprehensively investigated the infrastructure and optimal conditions of the citrate stabilized NPs of AuNPs (using trisodium citrate only) and AgNPs (trisodium citrate + Tannic acid) in a previous report by different techniques including SEM [31].

The most important characteristic features encountered in metal nanoparticles is electromagnetic resonances due to collective oscillations of the conduction electrons named 'Plasmon's', excited by light and leading to strong light scattering and absorption together with an enhancement of the local electromagnetic field. This mode for metals such as gold and silver fall in the visible spectral region which produces a size dependence of the dielectric function and consequently of the refractive index [47].

\subsection{In-vitro-assay}

In order to be useful for some biomedical application, the material should be tested for its cytotoxicity, for which the in-vitro cell viability assay is a promising strategy, which could provide convenient and reliable information about the cytocompatibility of the newly designed nanomaterials $[37,48]$.

Therefore, the biocompatibility of the prepared NPs was assessed by in-vitro assay of fibroblast cell line for $24 \mathrm{~h}$ and 3 days (Fig. 7). The cells grow very well in contact with all the three kinds of NPs tested (i.e. AgNPs, AuNPs and AuNP-PEG). Moreover, during $24 \mathrm{~h}$ incubation, the cells have smaller size and were less proliferated, as expected. After 3 days incubation the cells were larger in size and more proliferated (3days I). The growth was more pronounced in AuNPs and AuNP-PEG as can be seen more clearly from the lower magnification images (3-days II). Therefore, these bioengineered NPs showed good biocompability and can be used in various biotechnological areas in the future.

\section{Conclusions}

Here in this work we have synthesized silver and gold nanoparticles using two reducing agents tri-sodium citrate and gallic acid (GA) simultaneously to see the effect on the particle size, surface charge and cytotoxicity. The proposed approach is using green chemistry with convenient and safe reaction route for the preparation of such nanomaterials. These nanoparticles were analyzed by UV-visible spectroscopy, dynamic light scattering, and scanning electron microscopy. All of these characterization indicated that the particle size, size distribution and surface charge can be easily tuned by varying the GA concentration in the solution. Moreover, the PEGylation of them resulted in a larger particle size, while decreasing in zeta potential value. Furthermore, the in-vitro assay against human fibroblast cells (WI-38) indicated that all of them were completely safe for biomedical application. Hence, based on these obtained results it can be envisaged that both of these NPs have important applications in the nanobiotechnological area in the future. 

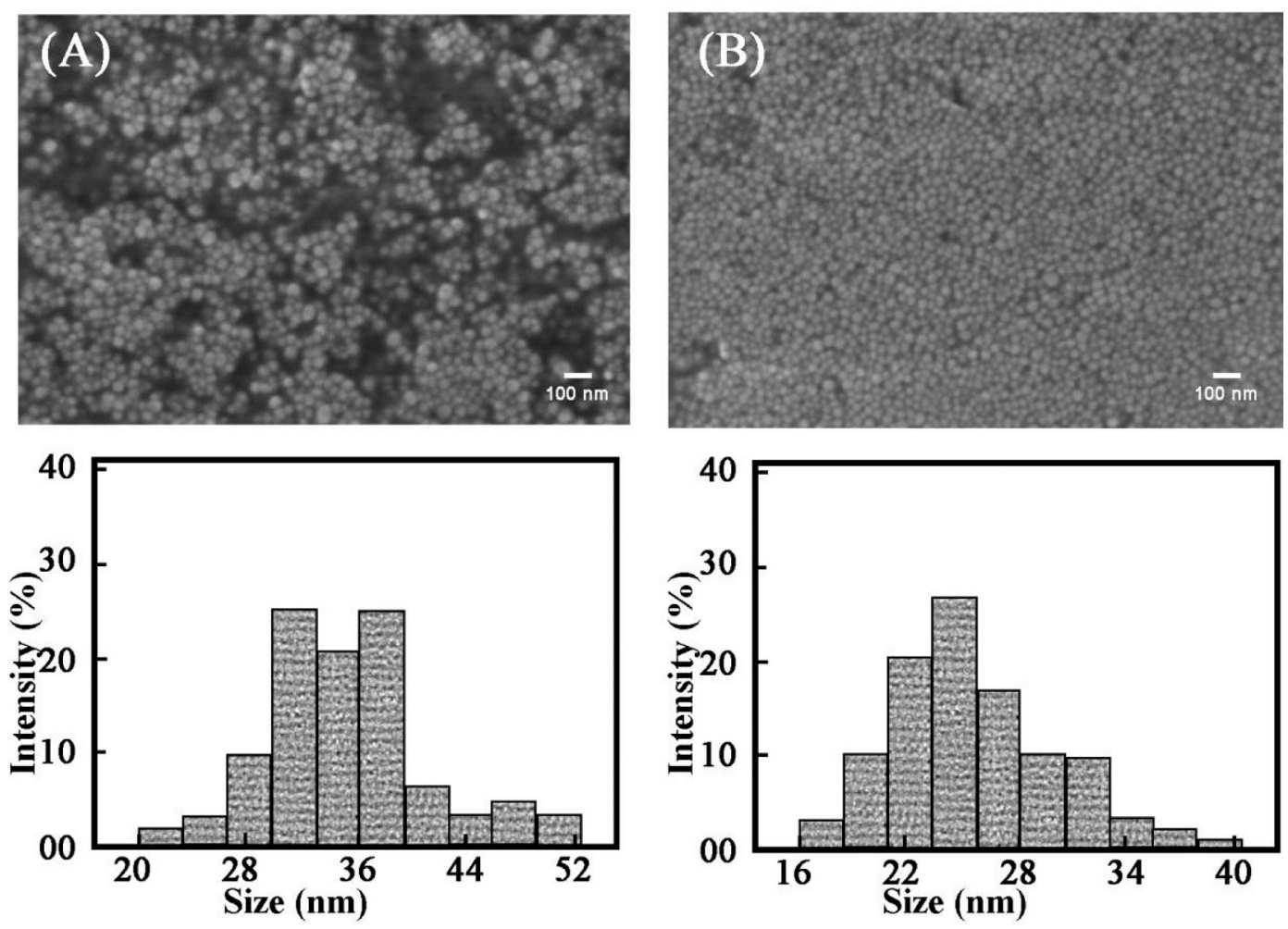

Fig. 6. SEM images of A) GA-AgNPs $35 \mathrm{~nm}$, B) GA-AuNPs $24 \mathrm{~nm}$ on glass substrate, scale bar $100 \mathrm{~nm}$.
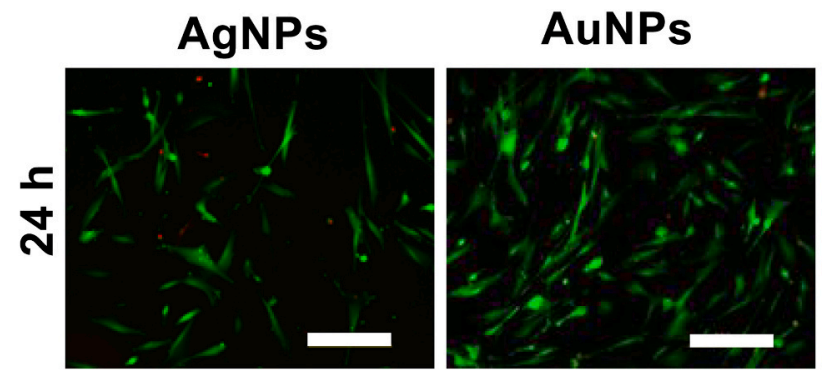

\section{AuNPs-PEG}
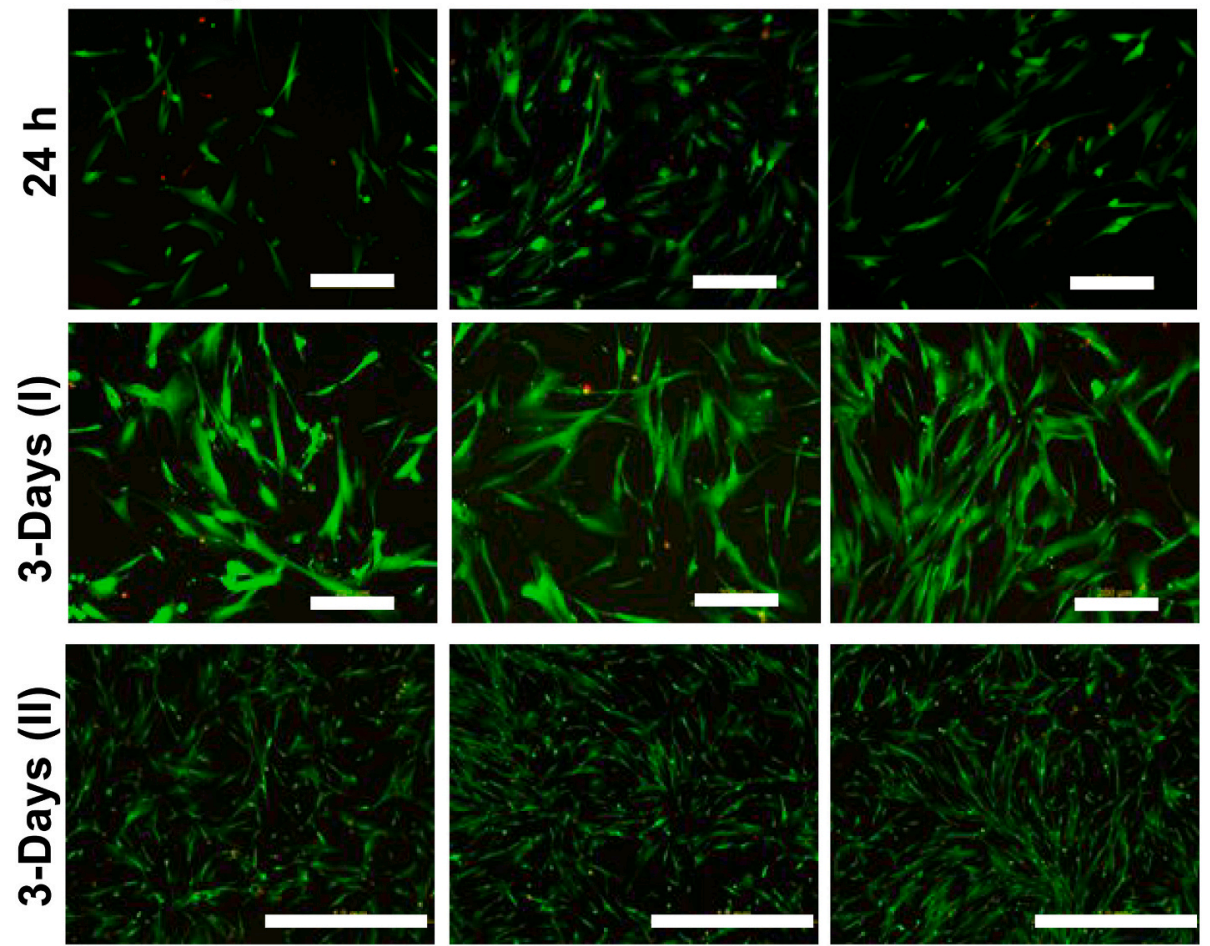

Fig. 7. Fibroblast cell culture with GA-AgNPs, GA-AuNPs and AuNP-PEG for $24 \mathrm{~h}$ and 3 days. Both $24 \mathrm{~h}$ and 3-days (I) using a small scale bar (magnified) $=200 \mu \mathrm{m}$, while 3-days (II) shows culture using larger scale bar $=1 \mathrm{~mm}$. 


\section{Declaration of competing interest}

The authors declare no competing financial interest for this work.

\section{Acknowledgments}

The authors acknowledge Tampere University of Technology (internal funding for postdoctoral researchers) and TEKES Finnish Funding Agency for Innovation project Human Spare Parts for financially supporting this work.

\section{References}

[1] F. Ahmad, L. Luo, X. Li, H. Huang, J. Zeng, Boosting fuel cell catalysis by surface doping of W on Pd nanocubes, Chin. J. Catal. 39 (2018) 1202-1209.

[2] X. Zhao, Y. Xing, L. Zhao, S. Lu, F. Ahmad, J. Zeng, Phosphorus-modulated cobalt selenides enable engineered reconstruction of active layers for efficient oxygen evolution, J. Catal. 368 (2018) 155-162.

[3] X. Li, X. Li, C. Liu, H. Huang, P. Gao, F. Ahmad, L. Luo, Y. Ye, Z. Geng, G. Wang, R. Si, C. Ma, J. Yang, J. Zeng, Atomic-level construction of tensile-strained pdfe alloy surface toward highly efficient oxygen reduction electrocatalysis, Nano Lett. 20 (2020) 1403-1409.

[4] R.C. Sanfelice, L.A. Mercante, A. Pavinatto, N.B. Tomazio, C.R. Mendonça, S.J.L. Ribeiro, L.H.C. Mattoso, D.S. Correa, Hybrid composite material based on polythiophene derivative nanofibers modified with gold nanoparticles for optoelectronics applications, J. Mater. Sci. 52 (2017) 1919-1929.

[5] S.H. Jeong, H. Choi, J.Y. Kim, T.W. Lee, Silver-based nanoparticles for surface plasmon resonance in organic optoelectronics, Part. Part. Syst. Charact. 32 (2015) 164-175.

[6] S. Nie, S.R. Emory, Probing single molecules and single nanoparticles by surfaceenhanced Raman scattering, Science 275 (1997) 1102-1106.

[7] Y. Yang, J. Shi, G. Kawamura, M. Nogami, Preparation of Au-Ag, Ag-Au core-shell bimetallic nanoparticles for surface-enhanced Raman scattering, Scr. Mater. 58 (2008) 862-865.

[8] F. Wang, D. Banerjee, Y. Liu, X. Chen, X. Liu, Upconversion nanoparticles in biological labeling, imaging, and therapy, Analyst. 135 (2010) 1839-1854.

[9] J. Jang, J. Ha, J. Cho, Fabrication of water-dispersible polyaniline-poly(4-styrenesulfonate) nanoparticles for inkjet-printed chemical-sensor applications, Adv. Mater. 19 (2007) 1772-1775.

[10] J.M. Pingarrón, P. Yáñez-Sedeño, A. González-Cortés, Gold nanoparticle-based electrochemical biosensors, Electrochim. Acta 53 (2008) 5848-5866.

[11] M. Arruebo, R. Fernández-Pacheco, M.R. Ibarra, J. Santamaría, Magnetic nanoparticles for drug delivery, Nano Today 2 (2007) 22-32.

[12] M. Hamidi, A. Azadi, P. Rafiei, Hydrogel nanoparticles in drug delivery, Adv. Drug Deliv. Rev. 60 (2008) 1638-1649.

[13] P. Štarha, D. Smola, J. Tuček, Z. Trávníček, Efficient synthesis of a maghemite/gold hybrid nanoparticle system as a magnetic carrier for the transport of platinumbased metallotherapeutics, Int. J. Mol. Sci. 16 (2015) 2034-2051.

[14] G. Sonavane, K. Tomoda, K. Makino, Biodistribution of colloidal gold nanoparticles after intravenous administration: effect of particle size, Colloids Surf. B: Biointerfaces 66 (2008) 274-280.

[15] M.R. Dewi, G. Laufersky, T. Nann, A highly efficient ligand exchange reaction on gold nanoparticles: preserving their size, shape and colloidal stability, RSC Adv. 4 (2014) 34217-34220.

[16] Z. Chen, X. Zhang, H. Cao, Y. Huang, Chitosan-capped silver nanoparticles as a highly selective colorimetric probe for visual detection of aromatic ortho-trihydroxy phenols, Analyst 138 (2013) 2343-2349.

[17] R. Arvizo, R. Bhattacharya, P. Mukherjee, Gold nanoparticles: opportunities and challenges in nanomedicine, Expert Opin. Drug Deliv. 7 (2010) 753-763.

[18] A. Luna-Velasco, J.A. Field, A. Cobo-Curiel, R. Sierra-Alvarez, Inorganic nanoparticles enhance the production of reactive oxygen species (ROS) during the autoxidation of 1-3,4-dihydroxyphenylalanine (1-dopa), Chemosphere 85 (2011) $19-25$.

[19] S.K. Balasubramanian, J. Jittiwat, J. Manikandan, C.N. Ong, L.E. Yu, W.Y. Ong, Biodistribution of gold nanoparticles and gene expression changes in the liver and spleen after intravenous administration in rats, Biomaterials 31 (2010) 2034-2042.

[20] M. Jeyaraj, G. Sathishkumar, G. Sivanandhan, D. MubarakAli, M. Rajesh, R. Arun, G. Kapildev, M. Manickavasagam, N. Thajuddin, K. Premkumar, A. Ganapathi, Biogenic silver nanoparticles for cancer treatment: an experimental report, Colloids Surf. B: Biointerfaces 106 (2013) 86-92.

[21] O.T. Jemilugba, E.H.M. Sakho, S. Paran, V. Mavumengwana, O.S. Oluwafemi, Green synthesis of silver nanoparticles using Combretum erythrophyllum leaves and its antibacterial activities, Colloid Interfac. Sci. Commun. 31 (2019) 100191.

[22] K. Jyoti, M. Baunthiyal, A. Singh, Characterization of silver nanoparticles synthesized using Urtica dioica Linn. leaves and their synergistic effects with antibiotics, J. Radiat. Res. Appl. Sci. 9 (2016) 217-227.
[23] L. Sherin, A. Sohail, U.S. Amjad, M. Mustafa, R. Jabeend, A.U. Hamid, Facile green synthesis of silver nanoparticles using Terminalia bellerica kernel extract for catalytic reduction of anthropogenic water pollutants, Colloid Interfac. Sci. Commun. 37 (2020) 100276

[24] A. Nour, M.T. Yarak, A. Lajevardi, Z. Rezaei, M. Ghorbanpour, M. Tanzifi, Ultrasonic-assisted green synthesis of silver nanoparticles using Mentha aquatica leaf extract for enhanced antibacterial properties and catalytic activity, Colloid Interfac. Sci. Commun. 35 (2020) 100252.

[25] N.G. Bastús, J. Comenge, V. Puntes, Kinetically controlled seeded growth synthesis of citrate-stabilized gold nanoparticles of up to $200 \mathrm{~nm}$ : size focusing versus ostwald ripening, Langmuir 27 (2011) 11098-11105.

[26] W.S. Cho, M. Cho, J. Jeong, M. Choi, B.S. Han, H.S. Shin, J. Hong, B.H. Chung, J. Jeong, M.H. Cho, Size-dependent tissue kinetics of PEG-coated gold nanoparticles, Toxicol. Appl. Pharmacol. 245 (2010) 116-123.

[27] S.J. Soenen, P. Rivera-Gil, J.M. Montenegro, W.J. Parak, S.C. De Smedt, K. Braeckmans, Cellular toxicity of inorganic nanoparticles: common aspects and guidelines for improved nanotoxicity evaluation, Nano Today 6 (2011) 446-465.

[28] A.K. Singh, O.N. Srivastava, One-step green synthesis of gold nanoparticles using black cardamom and effect of ph on its synthesis, Nanoscale Res. Lett. 10 (2015) 353.

[29] M. Wuithschick, A. Birnbaum, S. Witte, M. Sztucki, U. Vainio, N. Pinna, K. Rademann, F. Emmerling, R. Kraehnert, J. Polte, Turkevich in new robes: key questions answered for the most common gold nanoparticle synthesis, ACS Nano 9 (2015) 7052-7071.

[30] T. Zhu, K. Vasilev, M. Kreiter, S. Mittler, W. Knoll, Surface modification of citratereduced colloidal gold nanoparticles with 2-mercaptosuccinic acid, Langmuir 19 (2003) 9518-9525.

[31] M. Khan, S. Schuster, M. Zharnikov, Effect of humidity on electrical conductivity of pristine and nanoparticle-loaded hydrogel nanomembranes, J. Phys. Chem. C 119 (2015) 14427-14433.

[32] N.G. Bastús, F. Merkoçi, J. Piella, V. Puntes, Synthesis of highly monodisperse citrate-stabilized silver nanoparticles of up to $200 \mathrm{~nm}$ : kinetic control and catalytic properties, Chem. Mater. 26 (2014) 2836-2846.

[33] N. Nayeem, A. SMB, Gallic acid: a promising lead molecule for drug development, J. Appl. Pharmacol. 8 (2016) 1-4.

[34] N. Rattanata, S. Klaynongsruang, C. Leelayuwat, T. Limpaiboon, A. Lulitanond, P. Boonsiri, S. Chio-Srichan, S. Soontaranon, S. Rugmai, J. Daduang, Gallic acid conjugated with gold nanoparticles: antibacterial activity and mechanism of action on foodborne pathogens, Int. J. Nanomedicine 11 (2016) 3347.

[35] E. Detsri, P. Seeharaj, C. Sriwong, A sensitive and selective colorimetric sensor for reduced glutathione detection based on silver triangular nanoplates conjugated with gallic acid, Colloids Surf. A Physicochem. Eng. Asp. 541 (2018) 36-42.

[36] G. Ghodake, S. Shinde, A. Kadam, R.G. Saratale, G.D. Saratale, A. Syed, O. Shair, M. Alsaedi, D.Y. Kim, Gallic acid-functionalized silver nanoparticles as colorimetric and spectrophotometric probe for detection of $\mathrm{Al}^{3+}$ in aqueous medium, $\mathrm{J}$. Ind. Eng. Chem. 82 (2020) 243-253.

[37] M. Khan, J.T. Koivisto, T.I. Hukka, M. Hokka, M. Kellomäki, Composite hydrogels using bioinspired approach with in situ fast gelation and self-healing ability as future injectable biomaterial, ACS Appl. Mater. Interfaces 10 (2018) 11950-11960.

[38] D.Y. Kim, S. Shinde, G. Ghodake, Tuning stable and unstable aggregates of gallic acid capped gold nanoparticles using $\mathrm{Mg}^{2+}$ as coordinating agent, J. Colloid Interface Sci. 494 (2017) 1-7.

[39] M. Farrokhnia, S. Karimi, S. Askarian, Strong hydrogen bonding of gallic acid during synthesis of an efficient agnps colorimetric sensor for melamine detection via dis-synthesis strategy, ACS Sustain. Chem. Eng. 7 (2019) 6672-6684.

[40] J. Park, S.H. Cha, S. Cho, Y. Park, Green synthesis of gold and silver nanoparticles using gallic acid: catalytic activity and conversion yield toward the 4-nitrophenol reduction reaction, J. Nanopart. Res. 18 (2016) 166.

[41] D.Y. Kim, J. Suk Sung, M. Kim, G. Ghodake, Rapid production of silver nanoparticles at large-scale using gallic acid and their antibacterial assessment, Mater. Lett. 155 (2015) 62-64.

[42] D. Li, Z. Liu, Y. Yuan, Y. Liu, F. Niu, Green synthesis of gallic acid-coated silver nanoparticles with high antimicrobial activity and low cytotoxicity to normal cells, Process Biochem. 50 (2015) 357-366.

[43] A.K. Bhunia, T. Kamilya, S. Saha, Silver nanoparticle-human hemoglobin interface: time evolution of the corona formation and interaction phenomenon, Nano Converg. 4 (28) (2017) 1-12.

[44] N. Naiim, A. Boualem, C. Ferre, M. Jabloun, A. Jaloche, P. Ravier, Multiangle dynamic light scattering for the improvement of multimodal particle size distribution measurements, Soft Matter 11 (2015) 28-32.

[45] Y.J. Lee, K. Song, S.H. Cha, S. Cho, Y.S. Kim, Y. Park, Sesquiterpenoids from Tussilago farfara flower bud extract for the eco-friendly synthesis of silver and gold nanoparticles possessing antibacterial and anticancer activities, Nanomaterials 9 (2019) 819.

[46] S.H. Lee, B.H. Jun, Silver nanoparticles: synthesis and application for nanomedicine, Int. J. Mol. Sci. 20 (2019) 865.

[47] L.B. Scaffardi, J.O. Tocho, Size dependence of refractive index of gold nanoparticles, Nanotechnology 17 (2006) 1309.

[48] A. Pizzoferrato, G. Ciapetti, S. Stea, E. Cenni, C.R. Arciola, D. Granchi, Lucia, cell culture methods for testing biocompatibility, Clin. Mater. 15 (1994) 173-190. 